\title{
XQUESTION: Um aplicativo de perguntas e respostas para decisões estratégicas do professor durante uma aula
}

\author{
Adilmar C. Dantas ${ }^{1}$, Sara L. Melo ${ }^{1}$, Núbia F. Prado ${ }^{1}$, Márcia A. Fernandes ${ }^{1}$ \\ Eduardo K. Takahashi ${ }^{1}$, Marcelo Z. do Nascimento ${ }^{1}$ \\ ${ }^{1}$ Universidade Federal de Uberlândia (UFU) \\ Uberlândia - Minas Gerais - Brasil \\ \{adilmar, saraluziamelo, nubiafprd,marcia, ektakahashi,marcelo.nascimento\}@ufu.br
}

\begin{abstract}
Resumo. Este trabalho apresenta um aplicativo pessoal de respostas em tempo real, que tem com objetivo auxiliar professores elou tutores na tomada de decisões estratégicas durante uma aula. Na prática, os estudantes respondem questões formuladas pelos docentes no decorrer da aula, e simultaneamente o professor acessa suas respostas no mesmo instante, permitindo assim avaliar a necessidade ou não de estratégias ou revisões complementares para a compreensão do conteúdo em estudo. O mesmo foi testado e avaliado, tendo como principal vantagem o custo benefício sobre os dispositivos físicos.
\end{abstract}

\section{Cenário de Uso}

Atualmente, um dos principais problemas enfrentados pelos professores em seu ambiente escolar, está relacionado com o processo de ensino aprendizagem do aluno. Consequentemente, o docente procura métodos para melhorar os resultados de desempenho de seus discentes, mas encontra dificuldades na questão da tomada de decisões estratégicas no decorrer desse processo. Procurando solucionar esse problema, e utilizando como ferramenta a tecnologia, propomos o desenvolvimento de um aplicativo baseado em sistemas pessoais de resposta (personal response systems), também conhecidos por clickers ou response pulses, que são dispositivos tecnológicos semelhantes a controles remotos de TV, contendo, em geral, um teclado numérico e alguns botões de controle.

Eles permitem que o aluno responda, de forma rápida, questões apresentadas pelo professor durante uma aula expositiva-dialogada. O software de controle desse dispositivo possibilita ao professor obter rapidamente a estatística das respostas da sala, permitindo assim um feedback imediato da compreensão da matéria pelo aluno e, através disso, uma possível intervenção em relação ao andamento da aula.

Com o surgimento de novas tecnologias da informação surgiram inúmeras possibilidades para a educação. O próprio avanço da rede mundial de computadores e da rápida propagação da informação tornaram professores e alunos cada vez mais bem informados e conectados digitalmente [Mercado 2002], através de sistemas computacionais.

O uso desse tipo de tecnologia promove a aprendizagem ativa, pois utilizam-se de práticas pedagógicas, nas quais o aluno deixa de exercer somente o papel de "recebedor"de informações, uma vez que será inserido em um ambiente onde deve ser construtor dos seus conhecimentos de maneira proativa. Os benefícios proporcionados pela aprendizagem ativa são facilmente alcançados, principalmente no ensino superior [Martyn 2007], onde a maioria dos alunos faz uso dos mais modernos recursos tecnológicos para buscar 
o conhecimento. Entretanto, tais dispositivos apresentam os seguintes incômodos: são altamente dependentes do hardware, não exibem dados estatísticos na forma gráfica, exigem a transcrição manual dos dados para outra plataforma, não permitem a comunicação entre clickers de marcas diferentes, exigem manutenção permanente, e é necessária uma vigilância desses dispositivos para evitar perdas e danos.

Outro problema em que o aplicativo pode auxiliar de maneira significativa é em relação aos alunos com dificuldades de se expressar em discussões em público e consequentemente expor uma dúvida para a turma. Esse fato é facilmente contornado pela funcionalidade de não identificar o aluno durante a utilização, proporcionando a eles um momento agradável para expor sua dúvida sobre determinando assunto, de maneira simples e discreta.

Esse tipo de aplicação pode ser utilizada também com a finalidade de proporcionar aos estudantes uma aula interativa com o uso dessas tecnologias, que são cada vez mais presentes na sociedade moderna. Além disso, a elaboração de resumos e revisões para outros métodos de avaliação, como por exemplo, prova ou atividade avaliativa, seria um outro cenário onde o aplicativo poderia ser aproveitado, pois o professor e/ou tutor teria a possibilidade de obter um feedback atual dos alunos possibilitando que ele tome decisões estratégicas para a obtenção de melhores resultados nas avaliações.

\section{Desenvolvimento}

Para o desenvolvimento dos primeiros protótipos do Xquestion foi realizado um levantamento cuidadoso dos requisitos funcionais, que são as funções que o sistema deve oferecer em situações específicas e para a entrada de dados particulares, explicitamente o que a aplicação deve ou não fazer. Do mesmo modo, houve também uma investigação sobre os requisitos não funcionais, que é composto pelas funções extras oferecidas pela aplicação tais como manutenibilidade, usabilidade, desempenho e portabilidade [Sommerville and Sawyer 1997]. Todos os requisitos investigados são apresentados na tabela 1.

Tabela 1. Requisitos funcionais e não funcionais do aplicativo.

\begin{tabular}{|c|c|}
\hline Requisitos funcionais & Requisitos não funcionais \\
\hline $\begin{array}{l}\text { Cadastro de docente: } \mathrm{O} \text { aplicativo deve permitir o } \\
\text { cadastro de um ou mais docentes. }\end{array}$ & $\begin{array}{l}\text { Portabilidade: } \mathrm{O} \text { aplicativo deve permitir sua execução de } \\
\text { maneira transparente para o usuário em diferentes plataformas. }\end{array}$ \\
\hline $\begin{array}{l}\text { Cadastro de turmas: Permitir a inserção de turmas } \\
\text { para acesso dos alunos. }\end{array}$ & Mobilidade: Permitir a utilização por dispositivos móveis. \\
\hline $\begin{array}{l}\text { Cadastro de questões: Permitir a inserção de uma ou } \\
\text { mais questões para suas respectivas turmas. }\end{array}$ & Usabilidade: Possuir uma interface limpa e de fácil compreensão. \\
\hline $\begin{array}{l}\text { Clicker digital: Para a inserção das respostas dos } \\
\text { estudantes deve ser apresentado um dispositivo cliker } \\
\text { digital contendo quatro alternativas (A, B, C,D). }\end{array}$ & $\begin{array}{l}\text { Desempenho: A aplicação deve ser extremamente leve, permitindo } \\
\text { sua execução em situação limitantes como: internet lenta e dispositivos } \\
\text { móveis com pouca capacidade de armazenamento. }\end{array}$ \\
\hline $\begin{array}{l}\text { Relatórios visuais: Para o acompanhamento das respostas } \\
\text { deve ser exibido um relatório gráfico atualizado em tempo } \\
\text { real. }\end{array}$ & \\
\hline
\end{tabular}

O Xquestion foi projetado por meio de tecnologias atuais, permitindo uma maior usabilidade do sistema em ambientes educacionais. Para o seu desenvolvimento foram utilizadas tecnologias como Jquery Mobile, que consiste em um sistema de interface baseado no HTML5, possibilitando a criação de aplicações acessíveis [jQuery 2016], sendo possível sua execução em qualquer dispositivo. Esse tipo de aplicação é hoje denominado 
VI Congresso Brasileiro de Informática na Educação (CBIE 2017)

Anais dos Workshops do VI Congresso Brasileiro de Informática na Educação (WCBIE 2017)

como responsivos, possuindo a capacidade de se adaptar a qualquer plataforma. A Tabela 2 ilustra a execução do aplicativo desenvolvido, em diferentes dispositivos.

Para o armazenamento das informações do Xquestion foi utilizado o banco de dados Mysql, pelo fato desse banco de dados de código aberto ser o mais utilizado no mundo [Mysq1 2016], atualmente, sendo o líder para aplicações web, validando assim para o usuários um dos requisitos não funcionais, ao eliminar a necessidade de armazenamento interno no aparelho, pois as informações são processadas na nuvem.

Tabela 2. Teste de execução do aplicativo em navegadores e sistemas operacionais distintos

\begin{tabular}{lll}
\hline Navegador & Versões & Resultado \\
\hline Google Chrome & 58.0 & Funcional \\
Mozilla Firefox & 48.00 & Funcional \\
Internet Explorer & 11 & Com limitações \\
Safari & 5.1 .7 & Funcional \\
Android & 2.0 até 6.0 & Funcional \\
iOS & 5.0 até 8.0 & Funcional \\
\hline
\end{tabular}

Após o desenvolvimento da aplicação web responsiva foi criada as respectivas versões móveis para o sistema operacional Android, atualmente o sistema operacional móvel mais utilizado no mundo com núcleo baseado em Linux, desenvolvido pela empresa Google, e especialmente projetado para dispositivos móveis em geral [Deitel et al. 2013].

\section{Apresentação do software}

O Xquestion foi projetado para ser executado em todas as plataformas de maneira responsiva e síncrona nas seguintes versões: web, mobile Android e iOS. Por se tratar de uma aplicação híbrida, uma série de recomendações da web foram seguidas, conforme as especificações do HTML5 [W3C 2017], para que o aplicativo funcionasse sem limitações para os usuários. A inicialização do software é bem simples, primeiramente o usuário deve escolher em qual plataforma irá utilizá-lo. Para isso, deverá acessar o seguinte endereço http://xq.pushsistemas.com.br e definir se permanecerá na plataforma web ou se irá baixar alguma das versões mobile disponíveis, Android ou Ios, conforme a figura 1.

Posteriormente, caso o usuário seja um docente, ele deve fazer um cadastro rápido informando os seguintes dados: nome completo, e-mail institucional, selecionar sua instituição e por fim uma senha que será usada para acesso a área administrativa. Esses passos estão descritos na figura 2.

$\mathrm{Na}$ área administrativa o docente possui as seguintes funcionalidades enumeradas a seguir:

1) Cadastrar turmas: Funcionalidade onde professor e/ou tutor tem permissão para criar suas devidas turmas no qual o mesmo leciona.

2) Cadastrar questionamentos: Interface onde o associado a uma turma o professor e/ou tutor tem a possibilidade de criar as devidas questões a serem respondidas pelos alunos. 
VI Congresso Brasileiro de Informática na Educação (CBIE 2017)

Anais dos Workshops do VI Congresso Brasileiro de Informática na Educação (WCBIE 2017)

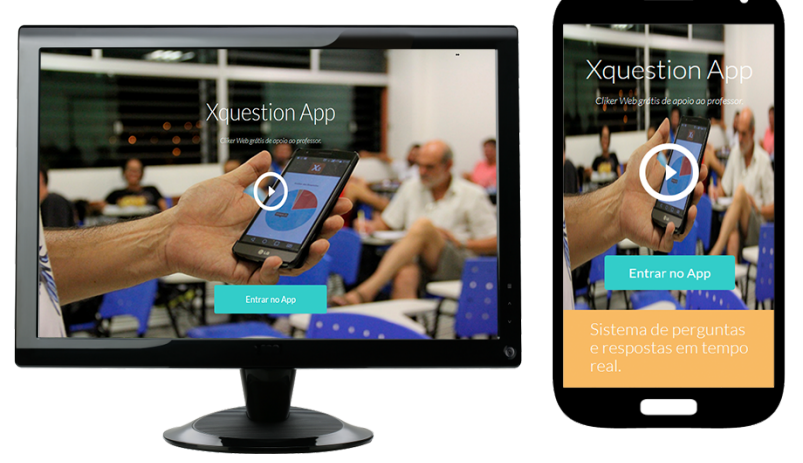

Figura 1. Passo 1 - Definido a plataforma de uso.

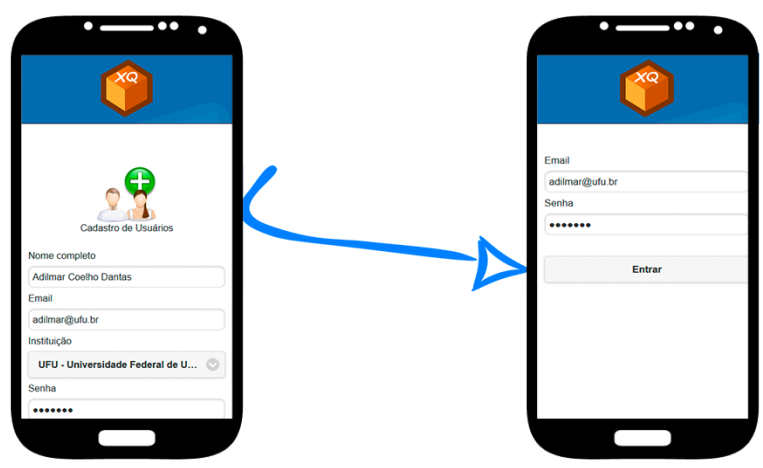

Figura 2. Passo 2 - Acessando a área do docente.

3) Gerar relatórios: Esta funcionalidade permite a visualização graficamente das respostas apresentadas pelos estudantes no aplicativo, de forma dinâmica.

4) Caixa de mensagens: Interface que permite que o professor visualize as mensagens enviadas a ele de forma privada pelos estudantes, apresentando dúvidas e comentários sobre uma determinada questão.

Todas essas funcionalidades descritas acima estão apresentadas na figura 3, a partir de captura do próprio aplicativo.

Os gráficos, que permite o professor acompanhar as respostas dos alunos, são atualizados em tempo real, a cada 10 segundos, de forma assíncrona e transparente para os usuários. No mesmo é possível visualizar também o número total de respostas e respostas por alternativas (A, B, C, D), conforme ilustrado na figura 4. As repostas dos alunos e os relatórios gráficos são armazenadas em banco de dados na nuvem, possibilitando assim que o professor acesse a qualquer momento esses relatórios, quando necessário, de maneira simples e direta.

Após o professor realizar todos os procedimentos para a criação das turmas e suas respectivas questões, os alunos devem acessar o aplicativo, selecionar uma das turmas disponíveis e realizar a resposta dos questionamentos através do cliker virtual, conforme ilustrado no fluxo de interfaces da figura 5. Através do aplicativo, o aluno consegue responder o questionamento ou enviar uma mensagem privada para o professor com suas possíveis dúvidas, em apenas três passos. 
VI Congresso Brasileiro de Informática na Educação (CBIE 2017)

Anais dos Workshops do VI Congresso Brasileiro de Informática na Educação (WCBIE 2017)

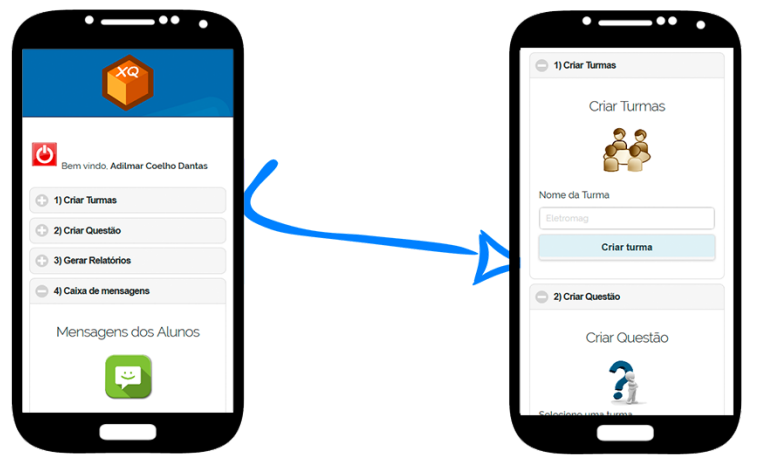

Figura 3. Passo 3 - Funcionalidades do professor e/ou tutor.

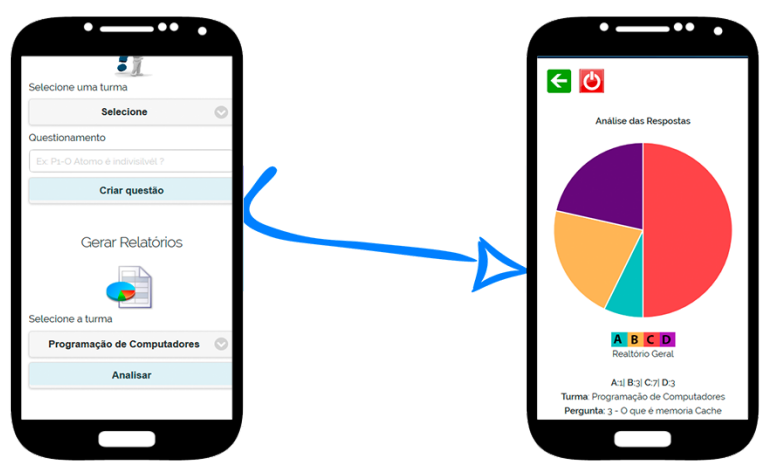

Figura 4. Passo 4 - Gerando relatórios em tempo real.
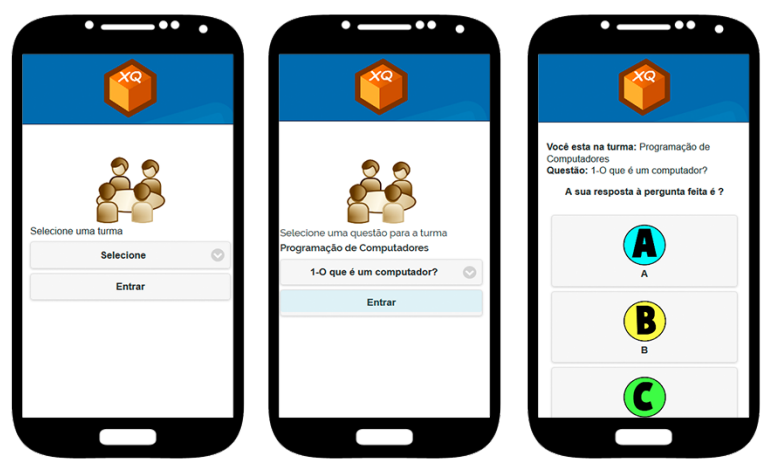

Figura 5. Passo 5 - Respondendo os questionamentos pelo cliker virtual.

Outra funcionalidade disponível no aplicativo, que dispositivos físicos não possuem, é a capacidade de um aluno entrar em contato com o professor através de mensagens instantâneas dentro do próprio aplicativo. Esse tipo de recurso é extremamente útil quando se tem pessoas com dificuldades de se expressar em grupo ou querem tirar uma dúvida de maneira particular.

Nessa interface o estudante entra com as informações como: qual questão está com dúvidas, nome do professor, seu nome e sua mensagem, que será encaminhada para o professor. No mesmo instante, o docente receberá uma notificação em sua área admi- 
VI Congresso Brasileiro de Informática na Educação (CBIE 2017)

Anais dos Workshops do VI Congresso Brasileiro de Informática na Educação (WCBIE 2017)

nistrativa, conforme exibido na figura 6.

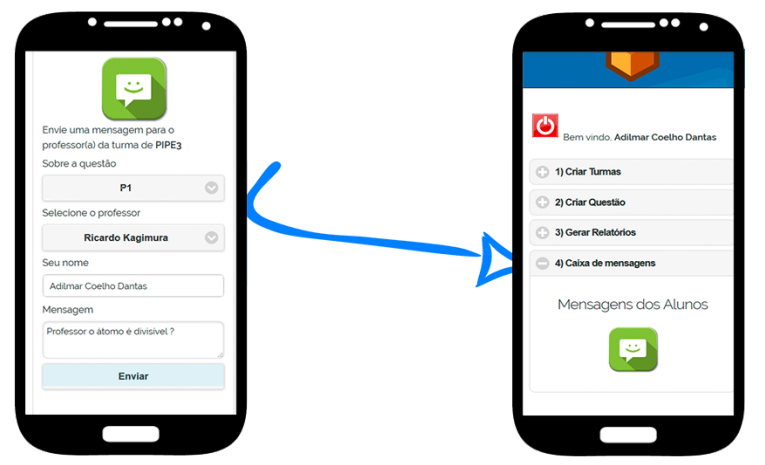

Figura 6. Funcionalidade para o envio de mensagens para o professor e/ou tutor.

\section{Considerações finais}

O uso do software Xquestion foi feito em uma turma de 51 estudantes da disciplina Introdução à Física, no curso de Licenciatura em Física, em uma Universidade Federal. Ao abordar o tema "Modelo Atômico de Bohr", os estudantes foram solicitados a responder 6 testes durante a aula, cujas respostas foram registradas pelos estudantes no aplicativo e avaliadas em tempo real pelo professor da disciplina.

As questões foram projetadas em tela durante a aula, de forma sequencial, e as respostas serviram de avaliação do grau de compreensão da sala sobre o tema. As respostas certas em porcentagem igual ou acima de $70 \%$ foram consideradas plenamente satisfatórias, enquanto $30 \%$ ou menos de respostas corretas foram consideradas muito problemáticas, onde alguma estratégia complementar foi adotada para procurar sanar as dúvidas dos alunos. As porcentagens intermediárias de acertos resultaram em discussões coletivas até a obtenção de um consenso. Cada teste foi analisado imediatamente após a obtenção das percentagens de acertos. Em relação a análise dos percentuais das respostas, obtivemos que 70,6\% dos alunos acertaram o teste 1 (resposta b), 84,1\% acertaram o teste 2 (resposta c), 64,4\% acertaram o teste 3 (resposta b), 77,1\% acertaram o teste 4 (resposta a), 39,5\% acertaram o teste 5 (resposta a) e 56,4\% acertaram o teste 6 (resposta c).

Assim, os conteúdos dos testes 1, 2 e 4 foram apenas generalizados, uma vez que apresentaram índices plenamente satisfatórios. Os testes 3, 5 e 6 receberam tratamentos mais cuidadosos por parte do professor e foram travadas discussões envolvendo análises das respectivas equações que fornecem as respostas, até que um consenso fosse obtido. Deve-se ressaltar que esse procedimento de identificação e discussão dos problemas na questão 3 contribuiu para aumentar o número de acertos na questão 4, cujo conteúdo era relacionado. O mesmo ocorreu nas questões 5 e 6 , demonstrando a importância da constatação imediata de problemas de aprendizagem dos estudantes.

Para uma melhor avaliação do aplicativo em diferentes contextos, o mesmo foi aplicado em uma turma com 27 estudantes da disciplina Programação de Computadores, do curso de Engenharia Aeronáutica, da Universidade Federal de Uberlândia. Ao abordar o tema Introdução a computação, os estudantes foram solicitados a responder 10 questões durante a aula teórica. Assim, as respostas foram registradas pelos estudantes no aplicativo e avaliadas em tempo real pelo professor da disciplina. Similarmente a disciplina 
VI Congresso Brasileiro de Informática na Educação (CBIE 2017)

Anais dos Workshops do VI Congresso Brasileiro de Informática na Educação (WCBIE 2017)

anterior, as questões foram projetadas em tela durante a aula, de forma sequencial, e as respostas dos alunos serviram de avaliação do grau de compreensão sobre o tema ministrado. A tabela 3 apresenta as questões aplicadas e respectivamente seus percentuais de acerto.

Tabela 3. Percentual de acertos por questão.

\begin{tabular}{lc}
\hline Questão & Percentual de Acerto \\
\hline O que é um computador? & $85,18 \%$ \\
O que é memória RAM & $37,03 \%$ \\
O que é memória Cache & $74,07 \%$ \\
O que é algoritmo? & $14,81 \%$ \\
Fortran encontra-se em qual paradigma & $88,88 \%$ \\
de programação? & $81.48 \%$ \\
O que é um compilador? & $85.18 \%$ \\
O que é um interpretador? & $74,07 \%$ \\
Quais as características da máquina & $96,29 \%$ \\
de Neumann? & $88,88 \%$ \\
Qual o nome do primeiro computador? & \\
Qual dispositivo não pertence a & memória secundária?
\end{tabular}

Por meio do aplicativo, as respostas certas com porcentagem igual ou acima de $70 \%$ foram consideradas plenamente satisfatórias para o professor, enquanto respostas com $30 \%$ ou menos de acerto foram consideradas muito problemáticas. Assim, ao verificar essa taxa de erro dos alunos, por meio do aplicativo, o professor aproveitou e efetuou uma revisão do conteúdo, relacionando as perguntas com alto índice de erros, com intuito de reforçar o conteúdo de aprendizagem. Para avaliar as principais vantagens do uso da tecnologia dos Clikers digitais, em relação aos Clikers físicos, construímos uma tabela 4 com as principais características dos dois dispositivos.

Tabela 4. Comparativo Software Cliker e Cliker Hardware

\begin{tabular}{|c|c|c|}
\hline Características & Cliker Software & Cliker Hardware \\
\hline Portabilidade & Sim. & Sim. \\
\hline Aquisição de Hardware & $\begin{array}{l}\text { Não, os hardwares são os próprios } \\
\text { dispositivos dos alunos e professor. }\end{array}$ & Sim um Cliker por aluno. \\
\hline Monitoramento do Hardware & $\begin{array}{l}\text { Não, existe esta necessidade pois cada } \\
\text { usuário é dono de seu aparelho. }\end{array}$ & $\begin{array}{l}\text { Sim, os aparelhos pertencem às instituições } \\
\text { de ensino, necessitando de monitoramento } \\
\text { durante o uso. }\end{array}$ \\
\hline Multiplataforma & $\begin{array}{l}\text { Sim, permite a execução em desktop, tablet, } \\
\text { e qualquer aparelho móvel. }\end{array}$ & $\begin{array}{l}\text { Não, na maioria das vezes existem apenas } \\
\text { alguns modelos que permitem uso em desktop. }\end{array}$ \\
\hline Personalização & $\begin{array}{l}\text { Sim, o professor pode personalizar seus } \\
\text { relatórios de acordo com suas necessidades. }\end{array}$ & $\begin{array}{l}\text { Não, geralmente os dados são apresentados de } \\
\text { uma única maneira sem muita personalização. }\end{array}$ \\
\hline Conexão & $\begin{array}{l}\text { Sim, para que seja possível acessar a aplicação } \\
\text { é necessário uma conexão com internet. }\end{array}$ & $\begin{array}{l}\text { Não, nenhum tipo de conexão externa é } \\
\text { necessária. }\end{array}$ \\
\hline
\end{tabular}

Observando a tabela notamos uma série de vantagens do uso do cliker digital, principalmente em relação ao custo benefício, o que torna a tecnologia desenvolvida acessível a maioria das instituições governamentais. As desvantagens levantadas estão sendo trabalhadas para serem sanadas em trabalhos futuros, garantido assim uma autonomia e flexibilidade do aplicativo em todas as situações. 
VI Congresso Brasileiro de Informática na Educação (CBIE 2017)

Anais dos Workshops do VI Congresso Brasileiro de Informática na Educação (WCBIE 2017)

\section{Referências}

Deitel, H. M., Deitel, A., and Morgano, M. (2013). Android para programadores: uma abordagem baseada em aplicativos. Bookman Editora.

jQuery (2016). Jquery mobile about@ONLINE.

Martyn, M. (2007). Clickers in the classroom: An active learning approach. Educause quarterly, 30(2):71.

Mercado, L. P. L. (2002). Novas tecnologias na educação: reflexões sobre a prática. UFAL.

Mysql (2016). Mysql about@ONLINE.

Sommerville, I. and Sawyer, P. (1997). Viewpoints: principles, problems and a practical approach to requirements engineering. Annals of software engineering, 3(1):101-130.

W3C (2017). The syntax, vocabulary and apis of html5@ ONLINE. 